TRANSACTIONS OF THE

AMERICAN MATHEMATICAL SOCIETY

Volume 363, Number 5, May 2011, Pages 2447-2465

S 0002-9947(2010)05005-6

Article electronically published on November 30, 2010

\title{
A COLDING-MINICOZZI STABILITY INEQUALITY AND ITS APPLICATIONS
}

\author{
JOSÉ M. ESPINAR AND HAROLD ROSENBERG
}

\begin{abstract}
We consider operators $L$ acting on functions on a Riemannian surface, $\Sigma$, of the form

$$
L=\Delta+V-a K .
$$

Here $\Delta$ is the Laplacian of $\Sigma, V$ a nonnegative potential on $\Sigma, K$ the Gaussian curvature and $a$ is a nonnegative constant.

Such operators $L$ arise as the stability operator of $\Sigma$ immersed in a Riemannian 3-manifold with constant mean curvature (for particular choices of $V$ and $a$ ). We assume that $L$ is nonpositive acting on functions compactly supported on $\Sigma$ and we obtain results in the spirit of some theorems of FischerColbrie-Schoen, Colding-Minicozzi and Castillon. We extend these theorems to $a \leq 1 / 4$. We obtain results on the conformal type of $\Sigma$ and a distance (to the boundary) lemma.
\end{abstract}

\section{INTRODUCTION}

A stable compact domain $\Sigma$ on a minimal surface in a Riemannian 3-manifold $M^{3}$ is one whose area cannot be decreased up to second order by a variation of the domain leaving the boundary fixed. Stable oriented domains $\Sigma$ are characterized by the stability inequality for normal variations $\psi N$ [20]

$$
\int_{\Sigma} \psi^{2}|A|^{2}+\int_{\Sigma} \psi^{2} \operatorname{Ric}_{\mathcal{M}^{3}}(N, N) \leq \int_{\Sigma}|\nabla \psi|^{2}
$$

for all compactly supported functions $\psi \in H_{c}^{1,2}(\Sigma)$. Here $|A|^{2}$ denotes the square of the length of the second fundamental form of $\Sigma, \operatorname{Ric}_{\mathcal{M}^{3}}(N, N)$ is the Ricci curvature of $\mathcal{M}^{3}$ in the direction of the normal $N$ to $\Sigma$ and $\nabla$ is the gradient w.r.t. the induced metric.

One writes the stability inequality in the form

$$
\left.\frac{d^{2}}{d t^{2}}\right|_{t=0} \operatorname{Area}(\Sigma(t))=-\int_{\Sigma} \psi L \psi \geq 0,
$$

where $L$ is the linearized operator of the mean curvature

$$
L=\Delta+|A|^{2}+\operatorname{Ric}_{\mathcal{M}^{3}} .
$$

Received by the editors December 10, 2008.

2000 Mathematics Subject Classification. Primary 53A10; Secondary 49Q05, 53C42.

Key words and phrases. Stability, conformal type, Distance Lemma.

The author was partially supported by Spanish MEC-FEDER Grant MTM2007-65249, and Regional J. Andalucía Grants P06-FQM-01642 and FQM325.

(C) 2010 American Mathematical Society
Reverts to public domain 28 years from publication 
In terms of $L$, stability means that $-L$ is nonnegative, i.e., that all its eigenvalues are nonnegative. $\Sigma$ is said to have finite index if $-L$ has only finitely many negative eigenvalues.

Since the stability inequality is derived from the second variation formula under normal variations of $\Sigma$, geometrically, $\Sigma$ has finite index if there is only a finite dimensional space of normal variations which strictly decrease the area.

In the 1970s and 1980s, this subject received an important number of contributions (see 1, 4, 6, 5, 7, 8, ), and even now it is a topic of interest (see [13, 14, for surveys).

D. Fischer-Colbrie and R. Schoen [6] studied stable surfaces by considering the nonnegativity of operators on a surface $\Sigma$ with a metric $g$ of the form

$$
L=\Delta+V-a K,
$$

where $\Delta$ and $K$ are the Laplacian and Gaussian curvatures associated to $g$, respectively, $a$ is a positive constant and $V$ is a nonnegative function. The main result of [6] for stable surfaces in three-manifolds is based on the following: For every complete metric on the disk, there exists a number $a_{0}$ depending on the metric satisfying $0 \leq a_{0}<1$, so that for $a \leq a_{0}$, there is a positive solution of $\Delta-a K$, and for $a>a_{0}$ there is no positive solution. Note that the existence of a positive solution of $\Delta-a K$ is equivalent to the nonpositivity of $L=\Delta-a K$ (see [6]).

Then a natural question was: What is the optimal $a_{0}$ ? M. do Carmo and C. K. Peng [4] proved (implicitly) that $a_{0} \geq 1 / 2$ for every complete metric on the disk. Years later, S. Kawai [10] (following ideas of A.V. Pogorelov [15]) stated that $a_{0} \geq 1 / 4$ for a metric with nonpositive curvature.

T. Colding and W. Minicozzi [3] introduced a new technique to study this type of operator based on the first variation formula for length and the Gauss-Bonnet formula. Using this technique they obtained a formula which, when $a>1 / 2$, gives quadratic area growth of the geodesic disks on the surface and the integrability of the potential $V$ at the same time (note that the stability operator can be realized with the right choice of $V$ ).

Recently, P. Castillon [2] used the ideas of Colding-Minicozzi to improve their result to $a>1 / 4$. Moreover, he answered the question of the optimal value of $a_{0}$ and proved the following:

Theorem. Let $\Sigma$ be a complete noncompact Riemannian surface. Set

$$
a_{0}=\sup \left\{a \in \mathbb{R}^{+} \text {: there exists a positive solution to } \Delta u-a K u=0 \text { on } \Sigma\right\} \text {. }
$$

If $a_{0}>1 / 4$, then $\Sigma$ is conformally equivalent to $\mathbb{C}$ or $\mathbb{C}^{*}=\mathbb{C}-\{0\}$.

This improvement was by an appropriate choice of radial cutoff functions. In fact, the same cutoff function has been considered in [14 to obtain an analogous formula to that of Colding-Minicozzi but for $a>1 / 4$.

Indeed, the value $a_{0}=1 / 4$ is critical since this is the value for the Poincare metric on the disk. Thus one cannot expect to have Castillon type results without other hypotheses. That will be the main line of this work, the study of these operators when $a \leq 1 / 4$ under other hypothesis on the area growth of the geodesic disks, a subject not studied until now, as far as we know.

The paper is organized as follows. In Section 2 we establish the notation that we will use. We develop in Section 3 an inequality in the spirit of Colding-Minicozzi for the quadratic form associated to the differential operator $L=\Delta+V-a K$, 
$a>0$. We then apply this for a specific choice of radial cutoff function defined on a geodesic disk on the surface, studying the behavior when the radius tends to infinity. We will see why we need some hypotheses on the area growth of the geodesic disks in the case that $a \leq 1 / 4$.

In Section 4 we consider the problem posed by D. Fischer-Colbrie and R. Schoen when $a \leq 1 / 4$ :

Theorem 4.1. Let $\Sigma$ be a complete Riemannian surface with $k-A A G$. Set

$$
a_{0}=\sup \left\{a \in \mathbb{R}^{+}: \text {there exists a positive solution to } \Delta u-a K u=0 \text { on } \Sigma\right\} \text {. }
$$

If $k<2$ and $0 \leq a_{0} \leq 1 / 4$, then $\Sigma$ is conformally equivalent to $\mathbb{C}$ or $\mathbb{C}^{*}$. If $k=2, \Sigma$ is parabolic with finite topology.

Here, $k-$ AAG means:

Definition 2.2. Let $\Sigma$ be a Riemannian surface. We say that $\Sigma$ has Asymptotic Area Growth of degree $k(k-\mathrm{AAG})$ if there exist positive constants $k, C \in \mathbb{R}^{+}$such that

$$
\lim _{r \longrightarrow \infty} \frac{\operatorname{Area}(D(p, r))}{r^{k}}=C, \forall p \in \Sigma .
$$

In Section 5 we obtain a Huber-type theorem. We prove

Theorem 5.1. Let $\Sigma$ be a complete Riemannian surface with $k-A A G$ and $0<a \leq$ 1/4. Suppose that $L=\Delta-a K$ is nonpositive on $\Sigma \backslash \Omega, \Omega$ a compact set. Then, if $k \leq 2, \Sigma$ is conformally equivalent to a compact Riemann surface with a finite number of points removed.

Theorem 5.2. Let $\Sigma$ be a complete noncompact parabolic Riemannian surface such that $\int_{\Sigma} K^{+}<+\infty$, with $K^{+}=\max \{K, 0\}$. Suppose that $L=\Delta-a K$ is nonpositive on $\Sigma$, where $a>0$. Then

- $K \in L^{1}(\Sigma)$, i.e., it is integrable. In fact, $0 \leq \int_{\Sigma} K \leq 2 \pi \chi(\Sigma)$.

- $\Sigma$ has quadratic area growth.

- $\Sigma$ is conformally equivalent either to the plane or to the cylinder.

We will apply these results in Section 6 to stable surfaces. We will be able to bound the distance of any point to the boundary. This is known as the Distance Lemma (see [18], [16] or [14] in the more general version, i.e., for $a>1 / 4$ and $V \equiv c>0$ some constant, and [1] for a sharp bound in space forms).

In fact, the authors gave an explicit bound of this distance in terms of $a$, when $a>1 / 4$, and $c>0$. Here we generalize this result for $0<a \leq 1 / 4$, giving the existence of some constant which bounds this distance.

Theorem 6.2. Let $\Sigma$ be a Riemannian surface possibly with boundary and $k-A A G$. Suppose that $L=\Delta+V-a K$ is nonpositive, where $V \geq c>0$ and $0<a \leq 1 / 4$. Then, there exists a positive constant $C$ such that

$$
\operatorname{dist}_{\Sigma}(p, \partial \Sigma) \leq C, \forall p \in \Sigma .
$$

In particular, if $\Sigma$ is complete with $\partial \Sigma=\emptyset$, then it must be topologically a sphere. In addition, we will control the growth of the integral of the potential (known for $a>1 / 4)$; that is,

Theorem 6.4. Let $\Sigma$ be a complete Riemannian surface satisfying $k-A A G, k \leq 2$. Suppose that $L=\Delta+V-a K$ is nonpositive, where $V \geq 0$ and $0<a \leq 1 / 4$. Then, $V \in L^{1}(\Sigma)$, i.e., $V$ is integrable. 
Moreover, if $\Sigma$ has $k-A A G$ with $k>2$, then for $2(b+1) \geq k$ we have

$$
\int_{D(s)} V \leq C s^{2 b}
$$

for some positive constant $C$.

Finally, we consider a problem posed in [6] for stable surfaces immersed in a three-manifold. In [6, Theorem 3], they proved: Let $N$ be a complete oriented 3-manifold of nonnegative scalar curvature. Let $\Sigma$ be an oriented complete stable minimal surface in $N$. If $\Sigma$ is noncompact, conformally equivalent to the cylinder and the absolute total curvature of $\Sigma$ is finite, then $\Sigma$ is flat and totally geodesic.

Also, they state [6, Remark 2]: We feel that the assumption of finite total curvature should not be essential in proving that the cylinder is flat and totally geodesic.

Using Theorem 5.2, we are able to partially answer this question.

Theorem 6.6. Let $N$ be a complete oriented 3-manifold of nonnegative scalar curvature. Let $\Sigma$ be an oriented complete stable minimal surface in $N$. If $\Sigma$ is noncompact, conformally equivalent to the cylinder and $\int_{\Sigma} K^{+}$is finite, then $\Sigma$ is flat and totally geodesic.

\section{Preliminaries}

We denote by $\Sigma$ a connected Riemannian surface, with Riemannian metric $g$, and possibly with boundary $\partial \Sigma$. Let $p_{0} \in \Sigma$ be a point of the surface and $D\left(p_{0}, s\right)$, for $s>0$, denote the geodesic disk centered at $p_{0}$ of radius $s$. We assume that $\overline{D\left(p_{0}, s\right)} \cap \partial \Sigma=\emptyset$. Moreover, let $r$ be the radial distance of a point $p$ in $D\left(p_{0}, s\right)$ to $p_{0}$. We write $D(s)=D\left(p_{0}, s\right)$.

We also denote

$$
\begin{aligned}
l(s) & =\operatorname{Length}(\partial D(s)), \\
a(s) & =\operatorname{Area}(D(s)), \\
K(s) & =\int_{D(s)} K, \\
\chi(s) & =\text { Euler characteristic of } D(s) .
\end{aligned}
$$

Moreover, we will need the following result due to K. Shiohama and M. Tanaka (see [19]) which follows from the first variation formula for length and the GaussBonnet formula.

Theorem 2.1. The function $l$ is differentiable almost everywhere, and we have

(1) for almost all $r \in \mathbb{R}$,

$$
l^{\prime}(r) \leq 2 \pi \chi(r)-K(r),
$$

(2) for all $0 \leq a<b$,

$$
l(b)-l(a) \leq \int_{a}^{b} l^{\prime}(r)
$$

Here, ' denotes the derivative with respect to $r$.

Let $L=\Delta+V-a K$ be a differential operator on $\Sigma$ acting on piecewise smooth functions with compact support, i.e., $f \in C_{0}^{\infty}(\Sigma)$, where $a>0$ is constant, $V \geq 0, \Delta$ and $K$ the Laplacian and Gauss curvatures associated to the metric $g$, respectively. 
The index form of these kinds of operators is

$$
I(f)=\int_{\Sigma}\left\{\|\nabla f\|^{2}-V f^{2}+a K f^{2}\right\},
$$

where $\nabla$ and $\|\cdot\|$ are the gradient and norm associated to the metric $g$. One has

$$
\int_{\Sigma} f L f=-I(f) .
$$

We will use the following condition on the area growth of $\Sigma$.

Definition 2.2. Let $\Sigma$ be a Riemannian surface. We say that $\Sigma$ has Asymptotic Area Growth of degree $k(k-\mathrm{AAG})$ if there exist positive constants $k, C \in \mathbb{R}^{+}$such that

$$
\lim _{r \rightarrow \infty} \frac{\operatorname{Area}(D(p, r))}{r^{k}}=C, \forall p \in \Sigma .
$$

Note that, by the Triangle Inequality, this condition does not depend on the point $p$.

\section{A Colding-Minicozzi Stability ineQuAlity}

Here, we will establish a general inequality for $I(f)$ (see (2.3) when $f$ is a radial function defined on a geodesic disk, following the method used by T. Colding and W. Minicozzi in [3]. The proof of this can be found in [2], but we include it here for the sake of completeness. The final formulation is slightly different from that of [2].

Lemma 3.1 (Colding-Minicozzi stability inequality). Let $\Sigma$ be a Riemannian surface possibly with boundary and $K \not \equiv 0$. Let us fix a point $p_{0} \in \Sigma$ and positive numbers $0 \leq \varepsilon<s$ such that $\overline{D(s)} \cap \partial \Sigma=\emptyset$. Let us consider the differential operator $L=\Delta+V-a K$, where $V \geq 0$ and $a$ is a positive constant, acting on $f \in H_{0}^{1,2}(\Sigma)$. Let $f: D(s) \longrightarrow \mathbb{R}$ be a nonnegative radial function, i.e., $f \equiv f(r)$, such that

$$
\begin{aligned}
& f(r) \equiv 1, \quad \text { for } r \leq \varepsilon, \\
& f(r) \equiv 0, \quad \text { for } r \geq s, \\
& f^{\prime}(r) \leq 0, \quad \text { for } \varepsilon<r<s .
\end{aligned}
$$

Then, the following holds:

$$
\begin{aligned}
I(f) \leq & 2 a\left(\pi G(s)-f_{-}^{\prime}(\varepsilon) l(\varepsilon)\right)-\int_{D(s)} V f(r)^{2} \\
& +\int_{\varepsilon}^{s}\left\{(1-2 a) f^{\prime}(r)^{2}-2 a f(r) f^{\prime \prime}(r)\right\} l(r),
\end{aligned}
$$

where

$$
\begin{aligned}
G(s):= & -\int_{0}^{s}\left(f(r)^{2}\right)^{\prime} \chi(r) \leq 1 \\
f_{-}^{\prime}(\varepsilon):= & \lim _{\substack{r \rightarrow \varepsilon\\
}} f^{\prime}(r) . \\
& \varepsilon<r
\end{aligned}
$$


Proof. Let us denote

$$
\alpha=\int_{D(s)}\|\nabla f\|^{2}, \beta=\int_{D(s)} K f^{2} .
$$

On the one hand, by the Co-Area Formula,

$$
\alpha=\int_{D(s)}\|\nabla f\|^{2}=\int_{\varepsilon}^{s} f^{\prime}(r)^{2} \int_{\partial D(r)} 1=\int_{\varepsilon}^{s} f^{\prime}(r)^{2} l(r) .
$$

On the other hand, by Fubini's Theorem and integrating by parts, we have

$$
\beta=\int_{0}^{s} f(r)^{2} \int_{\partial D(r)} K=\int_{0}^{s} f(r)^{2} K^{\prime}(r)=-\int_{0}^{s}\left(f(r)^{2}\right)^{\prime} K(r) .
$$

Now, by (2.1) and $\left(f(r)^{2}\right)^{\prime}=2 f(r) f^{\prime}(r) \leq 0$, we have

$$
-\left(f(r)^{2}\right)^{\prime} K(r) \leq\left(f(r)^{2}\right)^{\prime}\left(l^{\prime}(r)-2 \pi \chi(s)\right) .
$$

Integrating by parts and taking into account that $\int_{0}^{s}\left(f(r)^{2}\right)^{\prime}=-1$, we obtain

$$
\begin{aligned}
\beta & \leq \int_{0}^{s}\left(f(r)^{2}\right)^{\prime}\left(l^{\prime}(r)-2 \pi \chi(r)\right)=-2 \pi \int_{0}^{s}\left(f(r)^{2}\right)^{\prime} \chi(r)+\int_{0}^{s}\left(f(r)^{2}\right)^{\prime} l^{\prime}(r) \\
& =2 \pi G(s)+\int_{0}^{s}\left(f(r)^{2}\right)^{\prime} l^{\prime}(r)=2 \pi G(s)+\int_{\varepsilon}^{s}\left(\left(f(r)^{2}\right)^{\prime} l(r)\right)^{\prime}-\int_{\varepsilon}^{s}\left(f(r)^{2}\right)^{\prime \prime} l(r) \\
& =2 \pi G(s)-2 f_{-}^{\prime}(\varepsilon) l(\varepsilon)-\int_{\varepsilon}^{s}\left(f(r)^{2}\right)^{\prime \prime} l(r) .
\end{aligned}
$$

Thus, putting $\alpha$ and $\beta$ together,

$$
\begin{aligned}
\int_{D(s)}\left\{\|\nabla f\|^{2}+a K f^{2}\right\} & \leq 2 a\left(\pi \chi(s)-f^{\prime}(\varepsilon) l(\varepsilon)\right) \\
& +\int_{\varepsilon}^{s}\left\{(1-2 a) f^{\prime}(r)^{2}-2 a f(r) f^{\prime \prime}(r)\right\} l(r) .
\end{aligned}
$$

Now, we will work with the special radial function given by

$$
f(r)=\left\{\begin{array}{cc}
1 & r \leq s e^{-s}, \\
\left(\frac{\ln (s / r)}{s}\right)^{b} & s e^{-s} \leq r \leq s \\
0 & r \geq s
\end{array}\right.
$$

where $s>0, b \geq 1$ and $r$ is the radial distance of a point $p$ in $D(s)$ to $p_{0}$.

We summarize the properties of this function in the following result. 
Proposition 3.2. Let $a$ and $s$ be positive constants and $f:[0, s] \longrightarrow \mathbb{R}$ the function given by (3.2). Denote

$$
\begin{aligned}
\alpha & =1+b \frac{1-4 a}{2 a}, \\
g(r) & =\frac{\ln (s / r)}{s}, \\
\phi(r) & =\alpha-s g(r), \\
F(r) & =(1-2 a) f^{\prime}(r)^{2}-2 a f(r) f^{\prime \prime}(r) .
\end{aligned}
$$

Then, for $r \in\left(s e^{-s}, s\right)$, we have

$$
\begin{aligned}
& f^{\prime}(r)=-\frac{b}{s r} g(r)^{b-1} \leq 0, \\
& F(r)=2 a b \frac{g(r)^{2(b-1)}}{s^{2} r^{2}} \phi(r) .
\end{aligned}
$$

Moreover, if $\alpha>0$ and $s>\alpha+\delta>\alpha>0$ for some positive constant $\delta$, then the intervals

$$
\mathcal{I}_{1}=\left[s e^{-s}, s e^{-(\alpha+\delta)}\right], \mathcal{I}_{2}=\left[s e^{-(\alpha+\delta)}, s e^{-\alpha}\right], \mathcal{I}_{3}=\left[s e^{-\alpha}, s\right]
$$

are well defined and

$$
\begin{aligned}
& F_{\mid \mathcal{I}_{1}} \leq-2 \delta a b(\alpha+\delta)^{2(b-1)} e^{2(\alpha+\delta)} \frac{1}{s^{2(b+1)}} \\
& F_{\mid \mathcal{I}_{2}} \leq 0 \\
& F_{\mid \mathcal{I}_{3}} \leq 2 a b \alpha^{2 b-1} e^{2 \alpha} \frac{1}{s^{2(b+1)}}
\end{aligned}
$$

Proof. First, (3.7) and (3.8) are straightforward computations using the definitions of (3.3), (3.4), (3.5) and (3.6).

Let us assume that $\alpha>0$. Let $s>0$ be a positive number such that $s>\alpha+\delta$ for some $\delta>0$ fixed. Then

$$
e^{-s}<e^{-(\alpha+\delta)}<e^{-\alpha}<1,
$$

which means that the intervals $\mathcal{I}_{i}, i=1,2,3$, are well defined.

Since $g$ (given by (3.4) ) is a decreasing function, we have

$$
\begin{aligned}
\frac{\alpha+\delta}{s} & \leq g_{\mid \mathcal{I}_{1}} \leq 1 \\
\frac{\alpha}{s} & \leq g_{\mid \mathcal{I}_{2}} \leq \frac{\alpha+\delta}{s} \\
0 & \leq g_{\mid \mathcal{I}_{3}} \leq \frac{\alpha}{s}
\end{aligned}
$$


Thus,

$$
\begin{aligned}
\frac{(\alpha+\delta)^{2(b-1)} e^{2(\alpha+\delta)}}{s^{2 b}} & \leq\left(\frac{g(r)^{2(b-1)}}{r^{2}}\right)_{\mid \mathcal{I}_{1}} \leq \frac{e^{2 s}}{s^{2}} \\
\frac{\alpha^{2(b-1)} e^{2 \alpha}}{s^{2 b}} & \leq\left(\frac{g(r)^{2 b)}}{r^{2}}\right)_{\mid \mathcal{I}_{2}} \leq \frac{(\alpha+\delta)^{2(b-1)} e^{2(\alpha+\delta)}}{s^{2 b}} \\
0 & \leq\left(\frac{g(r)^{2(b-1)}}{r^{2}}\right)_{\mid \mathcal{I}_{3}} \leq \frac{\alpha^{2(b-1)} e^{2 \alpha}}{s^{2 b}},
\end{aligned}
$$

and $\phi$ (given by (3.5) ) satisfies

$$
\begin{aligned}
\alpha-s & \leq \phi_{\mid \mathcal{I}_{1}} \leq-\delta \\
-\delta & \leq \phi_{\mid \mathcal{I}_{2}} \leq 0 \\
0 & \leq \phi_{\mid \mathcal{I}_{3}} \leq \alpha .
\end{aligned}
$$

Hence, from (3.8),

$$
\begin{aligned}
& F_{\mid \mathcal{I}_{1}} \leq-2 \delta a b(\alpha+\delta)^{2(b-1)} e^{2(\alpha+\delta)} \frac{1}{s^{2(b+1)}}, \\
& F_{\mid \mathcal{I}_{2}} \leq 0 \\
& F_{\mid \mathcal{I}_{3}} \leq 2 a b \alpha^{2 b-1} e^{2 \alpha} \frac{1}{s^{2(b+1)}},
\end{aligned}
$$

as desired.

Given $0 \leq r_{1}<r_{2}$, we denote

$$
A\left(r_{1}, r_{2}\right)=a\left(r_{2}\right)-a\left(r_{1}\right),
$$

that is,

$$
A\left(r_{1}, r_{2}\right)=\int_{r_{1}}^{r_{2}} l(r)
$$

Also, we denote

$$
\mathcal{K}\left(r_{1}\right)=\min _{\left[0, r_{1}\right]}\{K(r)\} .
$$

Lemma 3.3. Let $\Sigma$ be a Riemannian surface possibly with boundary and $K \not \equiv 0$. Let us fix a point $p_{0} \in \Sigma$ and a positive number $s>0$ such that $\overline{D(s)} \cap \partial \Sigma=\emptyset$. Set $L=\Delta+V-a K$, where $V \geq 0$ and $a$ is a positive constant, acting on $f \in C_{0}^{\infty}(\Sigma)$. Given $b \geq 1$, let $\alpha$ be defined by (3.3). Then, if $\alpha>0$,

$$
\begin{aligned}
I(f) \leq 2 a & \left(\chi(s) \pi+b \frac{2 \pi-\mathcal{K}\left(s e^{-s}\right)}{s}\right)+\rho_{a, b}^{+}(\delta, s) \\
& -\left(\int_{D\left(s e^{-s}\right)} V+\int_{D(s) \backslash D\left(s e^{-s}\right)}\left(\frac{\ln (s / r)}{s}\right)^{2 b} V\right),
\end{aligned}
$$

where

$$
\rho_{a, b}^{+}(\delta, s)=2 a b \alpha^{2 b-1} e^{2 \alpha}\left(\frac{A\left(s e^{-\alpha}, s\right)}{s^{2(b+1)}}-\frac{\delta e^{2 \delta}}{\alpha}\left(1+\frac{\delta}{\alpha}\right)^{2(b-1)} \frac{A\left(s e^{-s}, s e^{-(\alpha+\delta)}\right)}{s^{2(b+1)}}\right) .
$$


Proof. We will use the function $f$ given by (3.2) in the equation (3.1) of Lemma 3.1 taking into account that $s e^{-s}$ plays the role of $\varepsilon$ in the formula, i.e., $\varepsilon=s e^{-s}$.

First, we will estimate the term $f^{\prime}(\varepsilon) l(\varepsilon)$ in (3.1).

Using (2.1) and (2.2), for any $\varepsilon>0$ we have

$$
l(\varepsilon) \leq \int_{0}^{\varepsilon} l^{\prime}(r) \leq 2 \pi \varepsilon-\min _{r \in[0, \varepsilon]}\{K(r)\} \varepsilon=(2 \pi-\mathcal{K}(\varepsilon)) \varepsilon .
$$

Also, by (3.7),

so, from (3.14) we obtain

$$
f^{\prime}\left(s e^{-s}\right)=-\frac{b}{s^{2} e^{-s}}
$$

$$
-f^{\prime}\left(s e^{-s}\right) l\left(s e^{-s}\right)=b \frac{1}{s} \frac{l\left(s e^{-s}\right)}{s e^{-s}} \leq b \frac{2 \pi-\mathcal{K}\left(s e^{-s}\right)}{s} .
$$

Thus,

$$
2 a\left(G(s) \pi-f^{\prime}\left(s e^{-s}\right) l\left(s e^{-s}\right)\right) \leq 2 a\left(G(s) \pi+b \frac{2 \pi-\mathcal{K}\left(s e^{-s}\right)}{s}\right) .
$$

Now, note that with the notation of Proposition 3.2, we have

$$
\int_{s e^{-s}}^{s}\left((1-2 a) f^{\prime}(r)^{2}-2 a f(r) f^{\prime \prime}(r)\right) l(r)=\int_{s e^{-s}}^{s} F(r) l(r) .
$$

Thus, from (3.9), (3.10) and (3.11),

$$
\begin{aligned}
& \int_{s e^{-s}}^{s} F(r) l(r) \leq \int_{\mathcal{I}_{1}} F(r) l(r)+\int_{\mathcal{I}_{2}} F(r) l(r)+\int_{\mathcal{I}_{3}} F(r) l(r) \\
& \quad \leq \int_{\mathcal{I}_{1}} F(r) l(r)+\int_{\mathcal{I}_{3}} F(r) l(r) \\
& \quad \leq 2 a b \alpha^{2 b-1} e^{2 \alpha} \frac{1}{s^{2(b+1)}} \int_{\mathcal{I}_{3}} l(r)-2 a b \delta(\alpha+\delta)^{2(b-1)} e^{2(\alpha+\delta)} \frac{1}{s^{2(b+1)}} \int_{\mathcal{I}_{1}} l(r) \\
& \quad=2 a b \alpha^{2 b-1} e^{2 \alpha}\left(\frac{A\left(s e^{-\alpha}, s\right)}{s^{2(b+1)}}-\frac{\delta e^{2 \delta}}{\alpha}\left(1+\frac{\delta}{\alpha}\right)^{2(b-1)} \frac{A\left(s e^{-s}, s e^{-(\alpha+\delta)}\right)}{s^{2(b+1)}}\right),
\end{aligned}
$$

that is,

$\int_{s e^{-s}}^{s} F(r) l(r) \leq 2 a b \alpha^{2 b-1} e^{2 \alpha}\left(\frac{A\left(s e^{-\alpha}, s\right)}{s^{2(b+1)}}-\frac{\delta e^{2 \delta}}{\alpha}\left(1+\frac{\delta}{\alpha}\right)^{2(b-1)} \frac{A\left(s e^{-s}, s e^{-(\alpha+\delta)}\right)}{s^{2(b+1)}}\right) ;$

hence, combining (3.15) and (3.16), we obtain (3.12).

Remark 3.4. Thus, it is clear from the above lemma that the behavior of $I(f)$ depends on the function $\rho^{+}$, which depends on the area growth of the surface.

The last results in this section are devoted to the asymptotic behavior of the function $\rho^{+}$under suitable conditions on the surface.

Lemma 3.5. Let $\Sigma$ be a Riemannian surface possibly with boundary satisfying $k-A A G$ and $K \not \equiv 0$. Given $b \geq 1$ and $a>0$, let $\alpha$ be defined by (3.3). Then, if $\alpha>0$, the asymptotic behavior of $\rho_{a, b}^{+}$, given by (3.13), as $s$ goes to infinity is

$$
\rho_{a, b}^{+}(\delta, s) \sim C^{+} \frac{s^{k}}{s^{2(b+1)}} \tilde{\rho}_{\alpha, k}^{+}(\delta),
$$


where

$$
\begin{aligned}
C^{+}(a, b, C) & =2 a b C \alpha^{2 b-1} e^{2 \alpha}, \\
\tilde{\rho}_{\alpha, k}^{+}(\delta) & =1-e^{-k \alpha}\left(1+e^{(2-k) \delta} \frac{\delta}{\alpha}\left(1+\frac{\delta}{\alpha}\right)^{2(b-1)}\right),
\end{aligned}
$$

and $C$ is the positive constant such that $a(s) \sim C s^{k}$.

Proof. We want to control the asymptotic behavior of $\rho^{+}$. Since $\Sigma$ has $k-$ AAG, this means that there exists $C>0$ such that

$$
a(s) \sim C s^{k}
$$

for $s$ large.

Hence

$$
\begin{aligned}
A\left(s e^{-s}, s e^{-(\alpha+\delta)}\right) & \sim C s^{k} e^{-k(\alpha+\delta)}, \\
A\left(s e^{-\alpha}, s\right) & \sim C s^{k}\left(1-e^{-k \alpha}\right)
\end{aligned}
$$

thus, from (3.13),

$$
\begin{aligned}
\rho_{a, b}^{+}(s, \delta) & \sim 2 a b C \alpha^{2 b-1} e^{2 \alpha} \frac{s^{k}}{s^{2(b+1)}}\left(1-e^{-k \alpha}-e^{2 \delta} e^{-k(\alpha+\delta)} \frac{\delta}{\alpha}\left(1+\frac{\delta}{\alpha}\right)^{2(b-1)}\right) \\
& =\left(2 a b C \alpha^{2 b-1} e^{2 \alpha}\right)\left(1-e^{-k \alpha}\left(1+e^{(2-k) \delta} \frac{\delta}{\alpha}\left(1+\frac{\delta}{\alpha}\right)^{2(b-1)}\right)\right) \frac{s^{k}}{s^{2(b+1)}} \\
& =C^{+} \frac{s^{k}}{s^{2(b+1)}} \tilde{\rho}_{\alpha, k}^{+}(\delta),
\end{aligned}
$$

as desired.

Remark 3.6. Let us note that the behavior of $\tilde{\rho}^{+}$depends strongly on the degree of the AAG. Moreover, we have that $\tilde{\rho}_{\alpha, k}^{+}(\delta)$ is a bounded function of $\delta \in \mathbb{R}^{+}$since it is continuous and

$$
\lim _{\delta \longrightarrow 0} \tilde{\rho}_{\alpha, k}^{+}(\delta)=1-e^{-k \alpha}=\lim _{\delta \longrightarrow+\infty} \tilde{\rho}_{\alpha, k}^{+}(\delta) .
$$

Thus there exists $\delta_{0}>0$ such that

$$
\rho_{\min }=\tilde{\rho}_{\alpha, k}^{+}\left(\delta_{0}\right)=\min _{\delta>0}\left\{\tilde{\rho}_{\alpha, k}^{+}\right\} .
$$

So, with this last remark in mind, we conclude

Corollary 3.7. Assuming the conditions of Lemma 3.5, if $\alpha>0$ and $2(b+1)>k$, then as $s \rightarrow+\infty$,

$$
\rho_{a, b}^{+}\left(\delta_{0}, s\right) \rightarrow 0,
$$

where $\delta_{0}$ is given in Remark 3.6 . 


\section{On a problem of D. Fischer-Colbrie and R. Schoen}

In [6], the authors proved: For every complete metric on the disk, there exists a number $a_{0}$ depending on the metric satisfying $0 \leq a_{0}<1$ so that for $a \leq a_{0}$ there is a positive solution of $\Delta-a K$, and for $a>a_{0}$ there is no positive solution. Here, $\Delta$ and $K$ denote the Laplacian and Gauss curvatures of the metric, respectively.

As we said in the introduction, P. Castillon 2] proved the following:

Theorem. Let $\Sigma$ be a complete noncompact Riemannian surface. Set

$$
a_{0}=\sup \left\{a \in \mathbb{R}^{+}: \text {there exists a positive solution to } \Delta u-a K u=0 \text { on } \Sigma\right\} .
$$

If $a_{0}>1 / 4$, then $\Sigma$ is conformally equivalent to $\mathbb{C}$ or $\mathbb{C}^{*}=\mathbb{C}-\{0\}$.

The method used for this is a formula as in Lemma 3.1 (to control the conformal type of the ends).

Moreover, in [2] and [14, it is shown that if $L_{a}=\Delta+a K \leq 0$ and $a>1 / 4$, then $\Sigma$ has at most quadratic area growth, i.e.,

$$
a(s) \leq C s^{2}
$$

for some positive constant $C$ and all $s>0$.

But, assuming some $k-\mathrm{AAG}$ on the surface we obtain the following (this is the first result we know of when $\left.a_{0} \in[0,1 / 4]\right)$.

Theorem 4.1. Let $\Sigma$ be a complete Riemannian surface with $k-A A G$. Set

$$
a_{0}=\sup \left\{a \in \mathbb{R}^{+} \text {: there exists a positive solution to } \Delta u-a K u=0 \text { on } \Sigma\right\} .
$$

If $k<2$ and $0 \leq a_{0} \leq 1 / 4$, then $\Sigma$ is conformally equivalent to $\mathbb{C}$ or $\mathbb{C}^{*}$. If $k=2$, $\Sigma$ is parabolic with finite topology.

Proof. Suppose that there exists $0<a \leq 1 / 4$ such that there exists a positive solution $u$ to $\Delta u-a K u=0$ on $\Sigma$. Then $L=\Delta-a K$ is nonpositive. On the one hand, consider the radial function $f(r)=(1-r / s), r \leq s$, in the equation (3.1) of Lemma 3.1 with $V \equiv 0$, then we obtain:

$$
0 \leq I(f) \leq 2 a \pi G(s)+\frac{(1-2 a)}{s^{2}} a(s) .
$$

On the other hand, assume that there exists $s_{0}$ so that for $s \geq s_{0}$ we have $\chi(s) \leq-M$, and then

$$
\begin{aligned}
G(s) & =-\int_{0}^{s}\left(f(r)^{2}\right)^{\prime} \chi(r)=-\int_{0}^{s_{0}}\left(f(r)^{2}\right)^{\prime} \chi(r)-\int_{s_{0}}^{s}\left(f(r)^{2}\right)^{\prime} \chi(r) \\
& \leq-\int_{0}^{s_{0}}\left(f(r)^{2}\right)^{\prime}+M \int_{s_{0}}^{s}\left(f(r)^{2}\right)^{\prime}=-\left(f\left(s_{0}\right)^{2}-f(0)^{2}\right)+M\left(f(s)^{2}-f\left(s_{0}\right)^{2}\right) \\
& =-(M+1) f\left(s_{0}\right)^{2}+1=-(M+1)\left(1-s_{0} / s\right)^{2}+1 .
\end{aligned}
$$

Thus, joining the above inequalities, we obtain

$$
0 \leq 2 a\left(1-(M+1)\left(1-s_{0} / s\right)^{2}\right)+\frac{1-2 a}{s^{2}} a(s) .
$$


Set

so

$$
T(s):=2 a\left(1-(M+1)\left(1-s_{0} / s\right)^{2}\right)+\frac{1-2 a}{s^{2}} a(s),
$$

$$
0 \leq \lim _{s \rightarrow+\infty} T(s)=-M+(1-2 a) \lim _{s \rightarrow+\infty} \frac{a(s)}{s^{2}} .
$$

If $k<2$, then $M \geq 0$, this means that $\Sigma$ is homeomorphic either to a plane or a cylinder.

If $k=2$, we have

$$
0 \leq \lim _{s \rightarrow+\infty} T(s)=-M+(1-2 a) C,
$$

for some positive constant $C$. Then, $M \leq(1-2 a) C$, that is, $\Sigma$ has finite topology.

It remains to prove that each end of $\Sigma$ is parabolic. This is clear since the area growth is quadratic.

Remark 4.2. Even with the AAG hypothesis, this result is sharp. R. Schoen pointed out to us that there exist hyperbolic surfaces with polynomial area growth greater than 2. Let us explain this. Consider the rotationally symmetric metric

$$
g=d r^{2}+\tau(r)^{2} d \theta
$$

on $\mathbb{R}^{2}$, with $\tau(r)=\frac{r^{1+\varepsilon}}{\varepsilon}$ for $\varepsilon>0$. Then, it is easy to see that the area of the geodesic disks is given by

$$
\operatorname{Area}(D(r))=\frac{2 \pi}{\varepsilon(1+\varepsilon)} r^{2+\varepsilon},
$$

and the Gaussian curvature is

$$
K=-\frac{\tau^{\prime \prime}}{\tau}=-\frac{1+\varepsilon}{r^{2}}
$$

Now, for $r$ large, we have

$$
K \leq-\frac{1+\varepsilon}{r^{2} \ln r}
$$

and $\tau$ is unbounded. Hence, using [12, Theorem 1], $\left(\mathbb{R}^{2}, g\right)$ is conformally hyperbolic.

\section{A Huber-type theOREm AND PARABOlicity}

Here we will establish a Huber-type theorem for surfaces with 2-AAG and $L=$ $\Delta-a K, 0<a \leq 1 / 4$. In fact, the proof follows from the work of P. Castillon [2].

Theorem 5.1. Let $\Sigma$ be a complete Riemannian surface with $k-A A G$ and $0<a \leq$ $1 / 4$. Suppose that $L=\Delta-a K$ is nonpositive on $\Sigma \backslash \Omega, \Omega$ a compact set. Then, if $k \leq 2, \Sigma$ is conformally equivalent to a compact Riemann surface with a finite number of points removed.

Proof. The main steps in [2, Theorem B] are controlling the topology and the area growth of the surface. Note that once we know that the surface has at most quadratic area growth, we control the conformal type of the ends. So, in the first item, as we are assuming at most $2-\mathrm{AAG}$, this last part is guaranteed. So, it remains to prove that the topology is finite.

We follow the proof of [2, Proposition 3.1]. 
Let $s_{0}, s_{1}>0$ such that $\Omega \subset D\left(s_{0}\right)$ and $s_{0}<s_{1}-1$. Define $f_{0}:\left[s_{1}-1, s_{1}\right] \longrightarrow \mathbb{R}$ by $f_{0}(r)=r-s_{1}+1$ and

$$
c_{a}=-a K\left(s_{1}\right)+\int_{D\left(s_{1}\right) \backslash D\left(s_{1}-1\right)}\left\{\left\|\nabla f_{0}(r)\right\|^{2}+a K f_{0}(r)^{2}\right\},
$$

which is a constant depending on $a$ and the metric.

Now, consider the radial function

$$
f(r)=\left\{\begin{array}{ccc}
f_{0}(r) & \text { for } & r \in\left[s_{1}-1, s_{1}\right] \\
1 & \text { for } & r \in\left[s_{1}, s_{2}\right] \\
\frac{s-r}{s-s_{2}} & \text { for } & r \in\left[s_{2}, s\right] \\
0 & & \text { elsewhere. }
\end{array}\right.
$$

Note that $f$ has compact support on $\Sigma \backslash D\left(s_{0}\right)$, so applying that $L$ is nonpositive, and following the computations of Lemma 3.1, we obtain

$$
0 \leq c_{a}+2 \pi a G(s)+\frac{2 a l\left(s_{2}\right)}{s-s_{2}}+\frac{1-2 a}{\left(s-s_{2}\right)^{2}} \int_{s_{2}}^{s} l(r),
$$

where

$$
G(s)=-\int_{s_{2}}^{s}\left(\left(\frac{s-r}{s-s_{2}}\right)^{2}\right)^{\prime} \chi(r) .
$$

Thus, since $\Sigma$ has at most $2-\mathrm{AAG}$, we have that

$$
c_{a}+\frac{2 a l\left(s_{2}\right)}{s-s_{2}}+\frac{1-2 a}{\left(s-s_{2}\right)^{2}} \int_{s_{2}}^{s} l(r) \longrightarrow C
$$

as $s \longrightarrow+\infty, C$ a positive constant.

If $\Sigma$ has infinite topology, then

$$
\liminf _{s \longrightarrow+\infty} \chi(s)=-\infty
$$

that is, we can take $s_{2}$ big enough so that $\chi(s) \leq-\frac{C+1}{2 a \pi}$ for all $s \geq s_{2}$, therefore

$$
G(s) \leq \frac{C+1}{2 a \pi} \int_{s_{2}}^{s}\left(\left(\frac{s-r}{s-s_{2}}\right)^{2}\right)^{\prime} \leq-\frac{C+1}{2 a \pi} .
$$

And so

$$
0 \leq 0 \leq c_{a}+2 \pi a G(s)+\frac{2 a l\left(s_{2}\right)}{s-s_{2}}+\frac{1-2 a}{\left(s-s_{2}\right)^{2}} \int_{s_{2}}^{s} l(r) \rightarrow-1
$$

as $s \rightarrow+\infty$, which is a contradiction. This completes the proof.

Theorem 5.2. Let $\Sigma$ be a complete noncompact parabolic Riemannian surface such that $\int_{\Sigma} K^{+}<+\infty$, with $K^{+}=\max \{K, 0\}$. Suppose that $L=\Delta-a K$ is nonpositive on $\Sigma$, where $a>0$. Then:

- $K \in L^{1}(\Sigma)$, i.e., it is integrable. In fact, $0 \leq \int_{\Sigma} K \leq 2 \pi \chi(\Sigma)$.

- $\Sigma$ has quadratic area growth.

- $\Sigma$ is conformally equivalent either to the plane or to the cylinder. 
Proof. The last two statements follow from the first one; let us explain this briefly.

Assume that $K \in L^{1}(\Sigma)$. Then (2.1) implies that

$$
l^{\prime}(r) \leq 2 \pi-K(r) \leq C,
$$

for some positive constant $C$, which means that $l(r) \leq C r$ from 2.2. Thus, $\Sigma$ has at most quadratic area growth. Now, either Theorem 4.1 for $a<1 / 4$ or [2, Theorem A] gives us the conformal type of the surface.

For a fixed point $p \in \Sigma$ and a sequence $s_{0}<s_{1}<s_{2}<\ldots \rightarrow+\infty$, let us consider the sequence of positive functions defined by

$$
\begin{array}{ccc}
\Delta f_{i}=0 & \text { on } & D\left(s_{i}\right) \backslash \overline{D\left(s_{0}\right)}, \\
f_{i}=1 & \text { on } & \overline{D\left(s_{0}\right),} \\
f_{i}=0 & \text { on } & \partial D\left(s_{i}\right) .
\end{array}
$$

Moreover, this sequence converges uniformly on compact subsets of $\Sigma$ to the constant function 1 and also is a monotone sequence by the Maximum Principle (see [13, Lemma 3.6]).

So, following [13, Theorem 10.1], using the boundary conditions and the fact that $f_{i}$ is harmonic on $D\left(s_{1}\right) \backslash D\left(s_{0}\right)$, we have

$$
\begin{aligned}
\int_{D\left(s_{i}\right) \backslash D\left(s_{0}\right)}\left\|\nabla f_{i}\right\|^{2} & =\int_{\partial D\left(s_{i}\right)} f_{i} \frac{\partial f_{i}}{\partial \eta}-\int_{\partial D\left(s_{0}\right)} f_{i} \frac{\partial f_{i}}{\partial \eta} \\
& =-\int_{\partial D\left(s_{0}\right)} \frac{\partial f_{i}}{\partial \eta},
\end{aligned}
$$

where $\frac{\partial f_{i}}{\partial \eta}$ is the outward pointing derivative.

Thus, using that $f_{i} \longrightarrow 1$ (uniformly on compact subsets), the right hand side must go to 0 as $s_{i} \longrightarrow+\infty$, that is,

$$
\int_{D\left(s_{i}\right) \backslash D\left(s_{0}\right)}\left\|\nabla f_{i}\right\|^{2} \longrightarrow 0, s_{i} \longrightarrow+\infty .
$$

Let us denote $K^{-}=\min \{0, K\}$ and $K^{+}=\max \{0, K\}$, so that $K=K^{-}+K^{+}$. Consider the sequence of monotone functions given by

$$
g_{i}^{+}=K^{+} f_{i}^{2}, \quad g_{i}^{-}=K^{-} f_{i}^{2}
$$

and note that for $i=1, g_{1}^{+}$and $g_{1}^{-}$are integrable on $\Sigma$.

Now, apply the nonpositivity of $L$ to the sequence $\left\{f_{i}\right\}$, i.e.,

$$
-a \int_{\Sigma} K f_{i}^{2} \leq \int_{\Sigma}\left\|\nabla f_{i}\right\|^{2}=\int_{D\left(s_{i}\right) \backslash D\left(s_{0}\right)}\left\|\nabla f_{i}\right\|^{2} .
$$

We write the left hand side of this inequality as

$$
-\int_{\Sigma} K f_{i}^{2}=-\int_{\Sigma} g_{i}^{+}-\int_{\Sigma} g_{i}^{-}
$$

By the Monotone Convergence Theorem for the sequences $\left\{g_{i}^{+}\right\}$and $\left\{g_{i}^{-}\right\}$(note that the limits could be infinite), we have

$$
\begin{aligned}
-\lim _{i \longrightarrow+\infty} \int_{\Sigma} K f_{i}^{2} & =-\lim _{i \longrightarrow+\infty} \int_{\Sigma} g_{i}^{+}-\lim _{i \longrightarrow+\infty} \int_{\Sigma} g_{i}^{-} \\
& =-\int_{\Sigma} K^{+}-\int_{\Sigma} K^{-}
\end{aligned}
$$

since $g_{i}^{ \pm} \longrightarrow K^{ \pm}$uniformly on compact sets. 
Thus, combining this with (5.2) and taking limits in (5.3), we have

$$
-\int_{\Sigma} K^{+}-\int_{\Sigma} K^{-} \leq 0
$$

but using that $\int_{\Sigma} K^{+}$is finite we obtain that $\int_{\Sigma} K^{-}$is finite; thus

$$
-\int_{\Sigma} K \leq 0
$$

since $a$ is a positive constant.

Now, since $\int_{\Sigma} K^{-}$is finite, the Cohn-Vosen inequality says that

$$
\int_{\Sigma} K \leq 2 \pi \chi(\Sigma)
$$

which means that

$$
0 \leq \int_{\Sigma} K \leq 2 \pi \chi(\Sigma)
$$

i.e., $K$ is integrable.

\section{Applications to stable surfaces}

In this section we study a nonpositive differential operator of the form

$$
L=\Delta+V-a K,
$$

where $V$ is a nonnegative function on $\Sigma$.

If $L f \leq 0$, then the quadratic form, $I(f)$, associated to $L$ is nonnegative on compactly supported functions, i.e., $I(f) \geq 0$. So, in this case, Lemma 3.3 can be rewritten as

Corollary 6.1. Let $\Sigma$ be a Riemannian surface possibly with boundary and $K \not \equiv 0$. Fix a point $p_{0} \in \Sigma$ and a positive number $s>0$ such that $\overline{D(s)} \cap \partial \Sigma=\emptyset$. Suppose that the differential operator $L=\Delta+V-a K$ is nonpositive on $C_{0}^{\infty}(\Sigma)$, where $V \geq 0$ and $a$ is a positive constant. Given $b \geq 1$, let $\alpha$ be defined by (3.3). With the notation of Lemma 3.3, if $\alpha>0$, then

$$
\int_{D\left(s e^{-s}\right)} V+\int_{D(s) \backslash D\left(s e^{-s}\right)}\left(\frac{\ln (s / r)}{s}\right)^{2 b} V \leq 2 a\left(G(s) \pi+b \frac{2 \pi-\mathcal{K}\left(s e^{-s}\right)}{s}\right)+\rho_{a, b}^{+}(\delta, s) .
$$

When $a>1 / 4$, we already know quadratic area growth and the integrability of the potential (see [2], 14] for $a>1 / 4$ or [3, [17, for $a>1 / 2$ ).

Another interesting consequence is that we are able to bound the distance of any point to the boundary. This is known as the Distance Lemma (see [18], 16] or [14, and 11] for a sharp bound in space forms).

Here, we will extend this result for $a \leq 1 / 4$.

Theorem 6.2. Let $\Sigma$ be a Riemannian surface with $k-A A G$ and possibly with boundary. Suppose that $L=\Delta+V-a K$ is nonpositive, where $V \geq c>0$ and $0<a \leq 1 / 4$. Then, there exists a positive constant $C$ such that

$$
\operatorname{dist}_{\Sigma}(p, \partial \Sigma) \leq C, \forall p \in \Sigma .
$$

In particular, if $\Sigma$ is complete with $\partial \Sigma=\emptyset$, then it must be topologically a sphere. 
Proof. Let us suppose that the distance to the boundary was not bounded. Then there would exist a sequence of points $\left\{p_{i}\right\} \in \Sigma$ such that $\operatorname{dist}^{\Sigma}\left(p_{i}, \partial \Sigma\right) \longrightarrow+\infty$. So, for each $p_{i}$ we could choose a real number $s_{i}$ such that $s_{i} \longrightarrow+\infty$ and $\overline{D\left(p_{i}, s_{i}\right)} \cap$ $\partial \Sigma=\emptyset$.

Let $\beta \in \mathbb{R}$ be a real number greater than one. Then

$$
\int_{D\left(s e^{-s}\right)} V+\int_{D(s) \backslash D\left(s e^{-s}\right)}\left(\frac{\ln (s / r)}{s}\right)^{2 b} V \geq c \frac{\beta^{2 b}}{s^{2 b}} a\left(s e^{-\beta}\right) .
$$

Now, choose $b>1$ such that $2(b+1) \geq k>2 b>2$. Thus, by (6.1) and the above inequality,

$$
c \frac{\beta^{2 b}}{s^{2 b}} a\left(s e^{-\beta}\right) \leq C+\rho_{a, b}^{+}\left(\delta_{0}, s\right)
$$

where $\delta_{0}$ is given in Remark 3.6.

Now, since $\Sigma$ has $k$-AAG and $k>2 b$, then for $s$ large enough we have

$$
c \frac{\beta^{2 b}}{s^{2 b}} a\left(s e^{-\beta}\right) \sim s^{k-2 b} \longrightarrow+\infty
$$

and from (3.17),

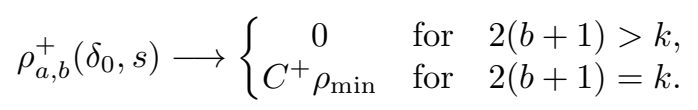

Thus, applying (6.2) to each disk $D\left(p_{i}, s_{i}\right)$, and bearing in mind that from (6.3) the left hand side of (6.2) goes to infinity, and (from (6.4) the right hand side remains bounded, we obtain a contradiction.

We still have to consider the case $k \leq 2$. Here, we consider a formula developed by Meeks-Pérez-Ros. This formula follows from Lemma 3.1 with the test function $f(r)=(1-r / s)^{b}$ for $r \in[0, s]$, that is, for $b \geq 1$,

$$
\int_{D(s)}(1-r / s)^{2 b} V \leq 2 a \pi+\frac{b(b(1-4 a)+2 a)}{s^{2}} \int_{0}^{s}(1-r / s)^{2 b-2} l(r) .
$$

Thus, for $b=1$ and the $k$-AAG, $k \leq 2$, of $\Sigma$, the right hand side of (6.5) goes to some positive constant as $s$ goes to infinity.

But, since $V \geq c>0$,

$$
\int_{D(s)}(1-r / s)^{2 b} V \geq c \frac{a(s / 2)}{4} .
$$

Thus, applying (6.5) to each disk $D\left(p_{i}, s_{i}\right)$, and bearing in mind that the left hand side of (6.5) goes to infinity and the right hand side remains bounded, we obtain a contradiction.

Note that we must be careful with the term $\mathcal{K}\left(s e^{-s}\right)$ in (6.1). Let us see that we do not need to worry about this term.

Let $p \in \Sigma$ be any point on the surface and consider the radial function $u(r)=$ $1-r / s$ defined on $D(p, s)$. Then, applying the nonpositivity of the operator $L=$ $\Delta+V-a K$, we have

$$
-a \int_{D(p, s)} u(r)^{2} K \leq \int_{D(p, s)}\|\nabla u\|^{2} \leq \frac{a(s)}{s^{2}} .
$$


Now, if $p$ is a point where $K(p)<0$ (note that we do not have to worry about points where the curvature is positive), we can choose $s>0$ small enough such that $K(q)<0$ for all $q \in D(p, s)$. Thus in this geodesic disk,

$$
-\frac{a}{4} \int_{D(p, s / 2)} K \leq-a \int_{D(p, s)} u(r)^{2} K \leq \frac{a(s)}{s^{2}},
$$

that is,

$$
-\int_{D(p, s / 2)} K \leq \frac{4 a(s)}{a s^{2}} .
$$

Taking into account that for $s$ small the area of $a(s)$ is almost Euclidean, we have

$$
\frac{4 a(s)}{a s^{2}} \longrightarrow C, s \longrightarrow 0
$$

for some positive constant $C$. So, we finally obtain that

$$
-\int_{D(p, s / 2)} K \longrightarrow C, s \longrightarrow 0,
$$

which means that

$$
\frac{\mathcal{K}\left(s e^{-s}\right)}{s} \longrightarrow 0, s \longrightarrow+\infty .
$$

Now, if $\Sigma$ is complete, then the estimate and the Hopf-Rinow Theorem imply that $\Sigma$ must be compact. Moreover, applying the operator $L_{a}$ to the test function 1 , we have

$$
a \int_{\Sigma} K \geq c \operatorname{Area}(\Sigma)
$$

which implies, by the Gauss-Bonnet Theorem, that $\chi(\Sigma)>0$.

As a consequence of this proof we have the following result.

Corollary 6.3. Under the hypothesis of Theorem 6.2, if $-L$ has finite index, then the distance of any point to the boundary is bounded. So, if the surface is complete, it must be compact and its Euler characteristic is positive.

Note that, following the above method, we can prove (for $a>1 / 4$ it is known)

Theorem 6.4. Let $\Sigma$ be a complete Riemannian surface satisfying $k-A A G, k \leq 2$. Suppose that $L=\Delta+V-a K$ is nonpositive, where $V \geq 0$ and $0<a \leq 1 / 4$. Then, $V \in L^{1}(\Sigma)$, i.e., $V$ is integrable.

Moreover, if $\Sigma$ has $k-A A G$ with $k>2$, then for $2(b+1) \geq k$ we have

$$
\int_{D(s)} V \leq C s^{2 b}
$$

for some positive constant $C$.

Proof. The case when $\Sigma$ has $k-\mathrm{AAG}, k \leq 2$, follows from formula (6.5), since then the right hand side goes to some constant, and we can bound the left hand side as

$$
\frac{1}{4} \int_{D(s / 2)} V \leq \int_{D(s)}(1-r / s)^{2} V
$$


The second case follows by using that

$$
\frac{(\ln 2)^{2 b}}{s^{2 b}} \int_{D(s / 2)} V \leq \int_{D\left(s e^{-s}\right)} V+\int_{D(s) \backslash D\left(s e^{-s}\right)}\left(\frac{\ln (s / r)}{s}\right)^{2 b} V
$$

and formula (3.17). So, putting this together with (6.1) we obtain the result.

Remark 6.5. Actually, the case $k \leq 2$ in the above result has been proven in [14] as well.

In [6. Theorem 3], they proved: Let $N$ be a complete oriented 3-manifold of nonnegative scalar curvature. Let $\Sigma$ be an oriented complete stable minimal surface in $N$. If $\Sigma$ is noncompact, conformally equivalent to the cylinder and the absolute total curvature of $\Sigma$ is finite, then $\Sigma$ is flat and totally geodesic.

Also, they state [6, Remark 2]: We feel that the assumption of finite total curvature should not be essential in proving that the cylinder is flat and totally geodesic.

So, using Theorem 5.2, we are able to partially answer this question.

Theorem 6.6. Let $N$ be a complete oriented 3-manifold of nonnegative scalar curvature. Let $\Sigma$ be an oriented complete stable minimal surface in $N$. If $\Sigma$ is noncompact, conformally equivalent to the cylinder and $\int_{\Sigma} K^{+}$is finite, then $\Sigma$ is flat and totally geodesic.

\section{ACKNOWLEDGEMENT}

The authors wish to thank J. Pérez and R. Schoen for their interesting comments and help during the preparation of this work.

\section{REFERENCES}

[1] L. Barbosa, M. do Carmo, On the size of a stable minimal surfaces in $\mathbb{R}^{3}$, Amer. Journ. Math. 98 (1976) no. 2, 515-528. MR0413172 (54:1292)

[2] P. Castillon, An inverse spectral problem on surfaces, Comment. Math. Helv. 81 (2006) no. 2, 271-286. MR2225628 (2007b:58042)

[3] T. Colding and W. Minicozzi, Estimates for parametric elliptic integrands, Internat. Math. Res. Notices 6 (2002), 291-297. MR 1877004 (2002k:53060)

[4] M. do Carmo and C. K. Peng, Stable minimal surfaces in $\mathbb{R}^{3}$ are planes, Bull. Amer. Math. Soc. 1 (1977), 903-906. MR546314 (80j:53012)

[5] D. Fischer-Colbrie, On complete minimal surfaces with finite Morse index in three manifolds, Invent. Math. 82 (1985), 121-132. MR808112 (87b:53090)

[6] D. Fischer-Colbrie and R. Schoen, The structure of complete stable minimal surfaces in 3manifolds of nonnegative scalar curvature, Comm. Pure Applied Math. 33 (1980), 199-211. MR562550 (81i:53044)

[7] R. Gulliver, Index and total curvature of complete minimal surfaces, Geometric measure theory and the calculus of variations (Arcata, Calif. 1984), Proc. Sympos. Pure Math. 44, Amer. Math. Soc., Providence, RI. (1986), 207-211. MR840274 (87f:53005)

[8] R. Gulliver, Minimal surfaces of finite index in manifolds of positive scalar curvature, Lecture Notes in Mathematics: Calculus of variations and partial differential equations (Trento, 1986) 1340, Springer, Berlin (1988), pp. 115-122. MR974606 (90c:53021)

[9] A. Huber, On subharmonic functions and differential geometry in the large, Comment. Math. Helv. 32 (1957), 13-72. MR0094452 (20:970)

[10] S. Kawai, Operator $\Delta-a K$ on surfaces, Hokkaido Math. Journal 17 (1988), 147-150. MR $945852(89 \mathrm{j}: 58149)$

[11] L. Mazet, Optimal length estimates for stable CMC surfaces in 3-space-forms, Preprint.

[12] J. Milnor, On deciding whether a surface is parabolic or hyperbolic, The Amer. Math. Monthly 84 no. 4 (1977), 43-46. MR0428232 (55:1257) 
[13] P. Li, Harmonic functions and applications to complete manifolds, XIV Escola de Geometria diferencial, IMPA, Rio de Janeiro (2006), ii+230pp. ISBN: 85-244-0249-0. MR 2369440 (2009a:53056)

[14] W. Meeks, J. Pérez and A. Ros, Stable constant mean curvature hypersurfaces, Preprint, available at http://www.ugr.es/local/jperez/papers/papers.htm.

[15] A. V. Pogorelov, On stability of minimal surfaces, Soviet Math. Dokl. 24 (1981), 274-276. MR630142 (83b:49043)

[16] A. Ros and H. Rosenberg, Properly embedded surfaces with constant mean curvature, Preprint.

[17] H. Rosenberg, Some recent developments in the theory of minimal surfaces, XXIV Coloquio Brasileiro de Matematica, Publicacoes Matematicas, IMPA, Rio de Janeiro (2003), 1-48. MR:2028922 (2005b:53015)

[18] H. Rosenberg, Constant Mean Curvature Surfaces in Homogeneously Regular 3-Manifolds, Bull. Aust. Math. Soc. 74 (2006), 227-238. MR2260491 (2007g:53009)

[19] K. Shiokama and M. Tanaka, The length function of geodesic parallel circles, Adv. Stud. Pure Math. (1993), 299-305. MR1274955 (95b:53054)

[20] R. Schoen and S.T. Yau, Harmonic maps and the topology of stable hypersurfaces and manifolds of nonnegative Ricci curvature, Comm. Math. Helv. 39 (1976), 333-341. MR0438388 $(55: 11302)$

Departamento de Geometría y Topología, Universidad de Granada, 18071 Granada, SPAIN

E-mail address: jespinar@ugr.es

Instituto de Matematica Pura y Aplicada, 110 Estrada Dona Castorina, Rio de JANEIRO 22460-320, BRAZIL

E-mail address: rosen@impa.br 